ISSN 1991-8631

Original Paper

http://indexmedicus.afro.who.int

\title{
Caractérisation physico-chimique de la gomme Sterculia de trois localités de la région de Tambacounda au Sénégal
}

\author{
Samba Arona Ndiaye SAMBA ${ }^{1 *}$, Mamoudou Abdoul TOURE ${ }^{2,3}$, Dame NIANG ${ }^{3}$ et \\ Yaye Kéne GASSAMA ${ }^{3}$
}

${ }^{1}$ École Nationale Supérieure d'Agriculture (ENSA), Université de Thiès, BP 967, Thiès, Sénégal. ${ }^{2}$ Institut Sénégalais de Recherches Agricoles - Route des hydrocarbures, Bel-Air, BP 3120 Dakar, Sénégal.

${ }^{3}$ Université Cheikh Anta Diop de Dakar, Département de Biologie Végétale, BP 5005, Dakar, Sénégal.

*Auteur correspondant, E-mail : bathie_samba@yahoo.fr, Tél. 221776528021

\section{RESUME}

La gomme sterculia est produite par Sterculia setigera Del. en Afrique. Ses propriétés physicochimiques changent suivant divers facteurs dont l'influence sur sa qualité a été peu étudiée. C'est dans cette perspective que l'humidité, le $\mathrm{pH}$, le pouvoir gonflant et la viscosité de cette gomme ont été évalués au Sénégal (Daoudi, Malem niani et Bala). Les résultats ont montré que son humidité varie en fonction du phénotype (écorce claire ou foncée). Elle a été plus élevée sur la gomme de la période sèche-chaude à Daoudi mais elle n'a pas changé suivant le site après un an de conservation. Son $\mathrm{pH}$ a varié en fonction de l'interaction période de saignée- couleur de l'écorce à Malem Niani alors qu'à Daoudi il a varié suivant la période de saignée seulement. A Malem, son gonflement a aussi varié suivant l'interaction période de saignée-couleur de l'écorce. Après un an de conservation (température ambiante), son pouvoir gonflant a diminué dans tous les sites. Sa viscosité a été 3,5 fois plus élevée à Bala qu'à Daoudi et Malem Niani. Ces résultats ont permis d'acquérir de nouvelles connaissances et d'identifier Bala comme ayant la meilleure qualité de gomme par rapport aux deux autres sites.

(C) 2012 International Formulae Group. All rights reserved.

Mots clés: Acidité, Humidité, pouvoir de gonflement, Sterculia setigera Del., Tambacounda, viscosité

\section{INTRODUCTION}

La gomme sterculia est produite par Sterculia setigera en Afrique, Sterculia urens et $S$. villosa en Inde, $S$. urceolata et $S$. foetida en Indonésie, S. caudata en Australie (Lô, 1996 ; Verbeken et al., 2003) et par certaines espèces du genre Cochlospermum de la famille des Bixaceae (Janaki et Sashidhar, 1998; Vinod et al., 2008; Vinod et al., 2010). La gomme de $S$. urens est autorisée à la consommation au même titre que les gommes de Acacia senegal, $S$. caudata, $S$. setigera et la gomme tragacanth des espèces asiatiques du genre Astragalus (Verbeken et al., 2003).

Les résultats d'Anderson et al. (1985) ont révélé une composition chimique similaire entre les gommes de $S$. urens (deux provenances d'Inde), $S$. villosa (deux provenances d'Inde) et $S$. setigera (quatre provenances $\mathrm{du}$ Soudan) mais avec une solubilité supérieure pour la gomme de $S$. setigera. Le mucilage de la gomme karaya est constitué d'acide acétique, de molécules de Dgalactose, de L-Rhamnose, d'acide D- 
Galacturonique et d'une faible quantité d'acide D-Glucuronique (Le Cerf et al., 1990 ; FAO, 1992; King et Richard, 1993 ; Lô, 1996 ; Babu et al., 2002a; Babu et al., 2002b; Verbeken et al., 2003).

Les propriétés physico-chimiques des gommes d'origine végétale peuvent changer en fonction du site d'origine, de l'espèce et des propriétés du sol (Islam et al., 1997 ; Idris et al., 1998; Karamalla et al., 1998). La viscosité de la gomme ( $S$. urens) varie en fonction de la période de récolte: elle est faible en période de pluies et élevée en période sèche. En Inde, le calibrage de la gomme karaya (S. urens) tient compte de plusieurs variables physico-chimiques dont la viscosité (Le Cerf, 1992 ; Bhattacharya et al., 2003 ; Kovel Foundation and International Resources Group, 2005 ).

Les solutions de gomme karaya sont sensibles à l'alcali et atteignent leur viscosité maximum à pH 8,5 (Verbeken et al., 2003). La même étude a montré que la gomme mbepp (345 cps) de $S$. setigera est plus visqueuse que la gomme karaya indienne (285 cps) produite par $S$. urens. La viscosité est aussi influencée par la granulométrie, la concentration en gomme, la température, la force ionique et le gradient de cisaillement (Lô, 1996 ; Babu et al., 2002b).

La gomme mbepp est largement consommée par les populations (Traoré, 1983 ; Bergeret et Ribot, 1990 ; Séne, 1994 ; Dieng, 1995; Lô, 1996; Henric, 2001 ; Johnson et al., 2005). Ces dernières conservent les récoltes de gomme pendant plusieurs mois dans des conditions peu propices, sans se soucier de sa détérioration. Or, le pouvoir gonflant, provoqué par la présence de groupements acétyles (Le Cerf et al., 1990), diminue à partir du quatrième mois après la récolte alors que la viscosité peut être maintenue pendant six à huit mois (Lô, 1996 ; Kovel Foundation and International Resources Group, 2005). Cette baisse de viscosité est plus accentuée lorsque la gomme est stockée sous forme de poudre que sous forme de granules brutes (Le Cerf et al., 1990 ; King et Richard, 1993 ; Babu et al., 2002b ; Verbeken et al., 2003) et elle est également plus élevée quand la gomme est dispersée en eau froide qu'en eau chaude (Dziezak, 1991).

L'étude de l'influence de la période, du site et de la durée de conservation de cette gomme sur certaines variables (humidité, acidité, gonflement, viscosité ...) a été peu étudiée en Afrique. En plus, certains exploitants affirment avoir identifié deux phénotypes de S. setigera (arbres à écorce claire et arbres à écorce foncée), d'où l'intérêt d'en tenir compte et d'étudier l'influence de la couleur de l'écorce sur les propriétés physicochimiques de la gomme.

Le chauffage des dispersions de gomme réduit de façon permanente la viscosité de la gomme karaya mais favorise l'augmentation de sa solubilité et permet la préparation des dispersions de $18 \%$, comparée à 4 - $5 \%$ en eau froide (Henric, 2001). Une diminution de la viscosité de la gomme karaya a aussi été observée avec l'addition d'électrolytes forts et d'acide ou d'alcali. Cette baisse de la viscosité est plus faible si la gomme est hydratée avant l'ajustement du pH (Whistler, 1993). La gomme karaya indienne donne un $\mathrm{pH}$ plus faible (4,4-4,7), une fois dispersée dans l'eau, que la gomme sterculia ( $\mathrm{pH} 4,5$ 5,2) africaine (Imeson, 1992).

L'étude comparative des gommes de $S$. urens (indien) et de $S$. setigera (africain) a révélé l'avantage que l'on peut tirer de l'utilisation de ces gommes, en raison de leur fort pouvoir de gonflement (Lô, 1996). La gomme sterculia possède un fort pouvoir hydrophile et peut absorber jusqu'à 100 fois son propre poids en eau (Verbeken et al., 2003).

En tout état de cause, la conservation des peuplements de $S$. setigera et la promotion de sa gomme devront passer par une meilleure 
connaissance des propriétés physicochimiques de cette dernière et par l'identification des facteurs pouvant les altérer.

\section{MATERIEL ET METHODES}

Humidité de la gomme mbepp (Sterculia setigera) en fonction des sites

La caractérisation de l'humidité de la gomme mbepp a été effectuée à partir de trois essais menés séparément dans trois sites: Bala (14 $01^{\prime}$ ' 63' 'Nord et $13^{\circ} 10^{\prime}$ 62' Ouest; altitude $65 \mathrm{~m}$ ), Daoudi ( $14^{\circ} 07^{\prime} 42^{\prime}$ ' Nord et $13^{\circ} 58^{\prime}$ 05' 'Ouest; altitude $33 \mathrm{~m}$ ) et Malem Niani $\left(13^{\circ} 56^{\prime} 00^{\prime \prime}\right.$ Nord et $14^{\circ} 18^{\prime} 00^{\prime \prime}$ Ouest; altitude $36 \mathrm{~m})$.

L'humidité de la gomme de Malem Niani a été étudiée suivant deux facteurs: la période de récolte de la gomme avec deux (2) niveaux (sèche - chaude et pluvieuse); et la couleur de l'écorce des troncs des arbres avec deux (2) niveaux (claire et foncée). Quatre traitements ont ainsi été administrés et chaque traitement a été répété vingt quatre (24) fois.

L'humidité de la gomme de Daoudi a été étudiée sur des échantillons récoltés en période sèche-chaude (mars à juin), en période de pluie (juillet à octobre) et en période sèchefraîche (novembre à février). Trois traitements ont ainsi été utilisés et chaque traitement a été répété trente six (36) fois. A Bala, l'humidité de la gomme n'a été mesurée que sur des échantillons récoltés en période sèche-chaude: un seul traitement a donc été utilisé et répété 72 fois.

Influence de la conservation et du site de récolte sur l'humidité de la gomme

Des échantillons de gomme collectés en période sèche-chaude ont été conservés à température ambiante pendant une année sous forme de granules (gomme nettoyée). Trois provenances de gomme, qui constituaient les traitements, ont été étudiées : Bala, Daoudi et
Malem Niani. Chaque traitement a été répété 36 fois.

L'étude des propriétés de la gomme mbepp a été effectuée avec une poudre obtenue après broyage (broyeur IK MF10 à 4000 tours/mn) de la gomme nettoyée. Le tamisage de la gomme a été réalisé en deux étapes: d'abord avec un tamis de $1 \mathrm{~mm}$ de maille, ensuite avec un deuxième tamis à mailles plus fines $(0,5 \mathrm{~mm})$.

Une balance électronique analyseur d'humidité (humidimètre) à infrarouge (Scalter) avec affichage simultané a été utilisée pour mesurer l'humidité de la gomme. La température de dessiccation a été fixée à $130{ }^{\circ} \mathrm{C}$. Pour chaque mesure d'humidité, $0,3 \mathrm{~g}$ (0,0001 g ; balance Mettler Toleso) de poudre de gomme posée sur une coupelle d'aluminium a été étudiée.

\section{Influence de la période de saignée et du phénotype sur le pH de la gomme}

Le pH de la gomme de Malem Niani a été étudié suivant deux facteurs: la période de récolte de la gomme avec deux (2) niveaux (sèche-chaude et pluvieuse); et la couleur de l'écorce (le phénotype) avec deux (2) niveaux (claire et foncée). Quatre (4) traitements ont ainsi été utilisés et chaque traitement a été répété douze (12) fois.

La caractérisation de l'acidité de la gomme de Daoudi a été effectuée sur des échantillons récoltés en période sèche-chaude, en période de pluie et en période sèchefraîche. Trois traitements ont été utilisés et chaque traitement a été répété dix huit (18) fois. A Bala, par contre, le $\mathrm{pH}$ de la gomme a été mesuré sur des échantillons récoltés en période sèche-chaude et chaque mesure a été répétée 36 fois.

\section{Influence du site de récolte sur le $\mathbf{p H}$ de la gomme \\ Trois provenances (ou sites de récolte) de gomme, qui constituaient les}


traitements, ont été étudiées : Bala, Daoudi et Malem Niani. Chaque traitement a été répété 36 fois. Les gommes ont été conservées à température ambiante pendant une année sous forme de granules.

Un gramme de poudre de gomme a été agité (magnétique) dans $100 \mathrm{ml}$ d'eau déminéralisée pendant $1 \mathrm{~h}$. Après $5 \mathrm{~min}$ de stabilisation (Lô, 1996), le pH de la suspension a été mesuré avec un pH-mètre Cybercan $\mathrm{pH} / \mathrm{Ion} 510$ possédant un écran avec affichage simultané.

Influence de la période de saignée et du phénotype sur le gonflement de la gomme

Le pouvoir gonflant de la gomme peut être défini comme la capacité pour une quantité de gomme donnée d'absorber un volume d'eau donné. Les populations qui exploitent la gomme rafrâichissent dans certaines localités (Malem Niani) les anciennes saignées pour effectuer une deuxième récolte de gomme. Pour étudier le pouvoir gonflant de la gomme entre les deux collectes (saignée et rafraîchissement), un essai à deux facteurs a été mis en place:

- Facteur 1: période de collecte avec quatre (4) niveaux (période pluvieuse 1 après saignée, période pluvieuse 2 après rafraîchissement, période sèche-chaude 1 après saignée et période sèche-chaude 2 après rafraîchissement);

- Facteur 2: couleur de l'écorce avec deux (2) niveaux (claire et foncée). L'unité expérimentale était représentée par un arbre. Huit (8) traitements ont ainsi été utilisés et chaque traitement a été répété douze (12) fois.

\section{Influence de la période de saignée sur le gonflement de la gomme}

La caractérisation du pouvoir gonflant de la gomme a été effectuée sur des échantillons récoltés à Daoudi en période sèche-chaude, en période de pluie et en période sèche-fraîche. Trois traitements, constitués par les différentes périodes, ont été utilisés et chaque traitement a été répété dix huit (18) fois.

\section{Influence du site de récolte sur le gonflement de la gomme \\ Trois provenances (ou sites de} récolte) de gomme, qui constituaient les traitements, ont été étudiées: Bala, Daoudi et Malem Niani. Chaque traitement a été répété trente six (36) fois. Les gommes ont été conservées à température ambiante pendant une année sous forme de granules. La méthode de la société pharmaceutique NORGINE (ex NORGAN, Dreux France) pour les gommes et adaptée à la gomme mbepp a été utilisée pour évaluer son pouvoir de gonflement (Lô, 1996). Un (1) gramme de poudre de gomme a été introduit dans une éprouvette de $1000 \mathrm{ml}(0,001$ l) remplie d'eau déminéralisée. Une agitation manuelle a été faite quatre fois dans la première heure, deux fois dans la deuxième heure, et enfin une fois par heure (h) durant $8 \mathrm{~h}$. Après un temps de repos de $18 \mathrm{~h}$, la lecture du gonflement a été effectuée par la mesure du volume occupé par le mélange y compris le mucilage, en pleine lumière et face à une vitre pour mieux visualiser la séparation du gel de l'eau.

\section{Viscosité de la gomme mbepp}

Des échantillons de gomme d'un an, récoltés en période sèche-chaude sur des sujets bons producteurs ont été utilisés pour l'étude de la viscosité. Un seul facteur a été étudié, le site de provenance de la gomme avec trois (3) niveaux: Bala, Daoudi et Malem Niani. Trois (3) traitements ont ainsi été utilisés et chaque traitement a été répété neuf (9) fois. La comparaison des viscosités des trois provenances de gomme a été effectuée sous une vitesse de cisaillement fixée à $0,35 \mathrm{~s}^{-1}$ et à la température de $35^{\circ} \mathrm{C}$.

La viscosité a été mesurée sur des solutions de gomme (1\%) préparées en 
mélangeant 2,5 g de poudre de gomme à 250 $\mathrm{ml}$ d'eau distillée dans un berlin de $500 \mathrm{ml}$; l'ensemble a été mis en agitation avec un barreau magnétique jusqu'à l'homogénéisation de la solution.

La viscosité a été mesurée avec un viscosimètre BRABENDER série VISCOTRON à cylindres coaxiaux dont l'un est fixe et l'autre mobile. L'appareil dispose d'un thermomètre à alcool qui sert à contrôler la température du bain-marie. La viscosité $(\eta)$ a été calculée à partir de la valeur lue $(\mathrm{S})$ sur le bloc de contrôle du viscosimètre en utilisant la formule:

Viscosité $(\eta)=([\mathrm{B} \times \mathrm{S} \times \mathrm{K}] / \mathrm{m})$ dans laquelle $S$ représente la lecture digitale; $\mathrm{D}$ la vitesse de cisaillement $=\mathrm{m} \mathrm{XX} \mathrm{s}^{-1} ; \tau$ la force de cisaillement $=\mathrm{B} \times \mathrm{S} \times \mathrm{Y}$ exprimée en $\mathrm{Pa}$; $\mathrm{m}$ ou rpm le nombre de révolution par minute $\left(\min ^{-1}\right) ; \mathrm{X}$ le facteur de cisaillement $=1,4 ; \mathrm{Y}$ le facteur de force (ressort pré calibré) = 0,0283 ; K la constante de calibration $=20,2$ et $\mathrm{B}=3$.

\section{Analyses statistiques}

Les variables mesurées sur la gomme sterculia dans cette série d'essais ont été l'humidité, le $\mathrm{pH}$, le pouvoir de gonflement et la viscosité. L'analyse statistique des données recueillies a été effectuée par une analyse de variance. $\mathrm{Au}$ préalable le test de Bartlett (1947) a permis de confirmer l'homogénéité des variances. Le test de Student NewmanKeuls a ensuite été utilisé pour identifier les traitements significativement différents.

\section{RESULTATS}

Influence de la période de récolte, du phénotype et du site sur l'humidité de la gomme

L'humidité de la gomme de Malem Niani a été plus élevée chez les arbres à écorce foncée $(16,82 \pm 0,28 \%)$ que chez les arbres à écorce claire $(15,98 \pm 0,28 \%$; p = 0,039; Figure 1).

L'humidité de la gomme de Daoudi a été plus élevée $(16,07 \pm 0,23 \%)$ pour les échantillons récoltés en période sèche-chaude suivis par ceux récoltés en période pluvieuse $(15,24 \pm 0,36 \%)$. Elle a été plus faible $(12,86$ $\pm 0,37 \%$ ) pour les échantillons collectés en période sèche-fraîche ( $\mathrm{p}=0,00001$; Figure 2). En période sèche-chaude, l'humidité de la gomme de Bala a été de 17,22 $\pm 2,47 \%$. L'humidité n'a pas varié $(17,96 \pm 0,58 \% ; 17$, $82 \pm 0,15 \%$ et $17,42 \pm 0,40 \%$, respectivement à Bala, Daoudi et Malem niani) entre les trois provenances de gomme après un an de conservation $(p=0,0720)$.

\section{Influence de la période de saignée et du phénotype sur le pH de la gomme}

Le $\mathrm{pH}$ de la gomme de Malem Niani a varié en fonction de l'interaction entre la période de saignée et la couleur de l'écorce ( $p$ $=0,026)$. Pour les arbres à écorce claire, l'acidité a été plus faible en période sèchefraîche $(6,47 \pm 0,09)$ et plus élevée en période sèche-chaude et en période pluvieuse $(5,31 \pm$ 0,10 en moyenne pour ces deux dernières; Figure 3). La même tendance a été constatée avec les arbres à écorce foncée avec un pH plus élevé en période sèche-fraîche $(6,05 \pm$ $0,01)$ et plus faible en période sèche-chaude et en période de pluies $(5,38 \pm 0,10$ en moyenne pour ces deux périodes ; Figure 3).

A Daoudi, l'acidité de la gomme récoltée a varié en fonction de la période de saignée ( $\mathrm{p}<0,00001)$ : elle a été plus élevée en période sèche-chaude $(5,04 \pm 0,032)$ qu'en période sèche-fraîche $(5,26 \pm 0,05)$. En période pluvieuse elle a encore été plus faible $(5,67 \pm 0,043$; Figure 4). La gomme de Bala a cependant présenté une acidité plus élevée en période sèche-chaude $(\mathrm{pH}=4,95 \pm 0,01 ; \mathrm{n}=$ 26) que celle de Daoudi. 
Influence du site de récolte sur le $\mathbf{p H}$ de la gomme

L'acidité de la gomme récoltée en période sèche-chaude a varié en fonction de son origine après un an de conservation $(\mathrm{p}<$ 0,00001). Elle a été plus élevée à Malem Niani $(5,53 \pm 0,07)$ et plus faible à Daoudi et Bala sans toutefois présenter des différences significatives entre ces dernières origines $(6,47 \pm 0,36$ par arbre ; Figure 5).

Influence de la période de saignée et de la couleur de l'écorce sur le gonflement

A Malem Niani, le gonflement a varié en fonction de l'interaction entre la période de saignée et la couleur de l'écorce ( $\mathrm{p}=0,039)$. En période de pluies, le gonflement de la gomme a été relativement faible et sans différence significative entre les arbres à écorce foncée et les arbres à écorce claire, pour une moyenne de 439,35 \pm 76,5 $\mathrm{ml}$. Bien que plus élevé en période sèche-chaude (saignée et rafraîchissement), le gonflement n'a pas varié entre les deux phénotypes avec une moyenne de $1000 \pm 75,5 \mathrm{ml}$.

Par contre, en période pluvieuse, après rafraîchissement des anciennes saignées, le pouvoir gonflant du phénotype foncé a été plus élevé $(437,5 \pm 76,5 \mathrm{ml})$ que celui du phénotype clair (120,4 $\pm 83,8 \mathrm{ml}$; Figure 6). Par contre, à Daoudi, pour chaque période de saignée, le gonflement de la gomme a été en moyenne de $1000 \pm 0,00 \mathrm{ml}(\mathrm{p}<0,00001)$. Le pouvoir de gonflement de cette provenance de gomme a donc été plus constant.

\section{Influence du site de récolte sur le gonflement de la gomme}

Après un an de conservation (température ambiante), le pouvoir gonflant de la gomme récoltée en période sèche-chaude $(1000 \mathrm{ml})$ a diminué dans tous les sites $(\mathrm{p}<$ 0,00001). Ainsi, le gonflement de la gomme de Bala a été réduit à $276 \pm 22,2 \mathrm{ml}$, celui de Daoudi à 126,54 $\pm 17,4 \mathrm{ml}$ et celui de Malem Niani à 50,55 $\pm 18,9 \mathrm{ml}$ (Figure 7).

\section{Viscosité de la gomme mbepp}

La viscosité de la gomme mbepp a été plus élevée ( $\mathrm{p}<0,00001)$ à Bala $(9913 \pm 515$ $\mathrm{mPa}$ ) qu'à Daoudi et Malem Niani, sans pour autant être significativement différente entre les deux derniers sites (en moyenne $2872 \pm$ $515 \mathrm{mPa}$; Figure 8).

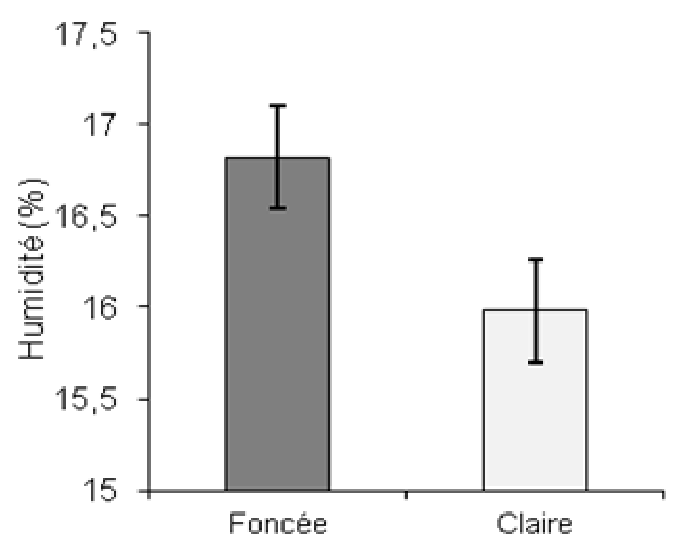

Figure 1: Influence de la couleur de l'écorce (Foncée, Claire) sur l'humidité (\%) de la gomme de $S$. setigera récoltée à Malem niani $(F=4,40 ; p=0,039 ; n=80)$. 


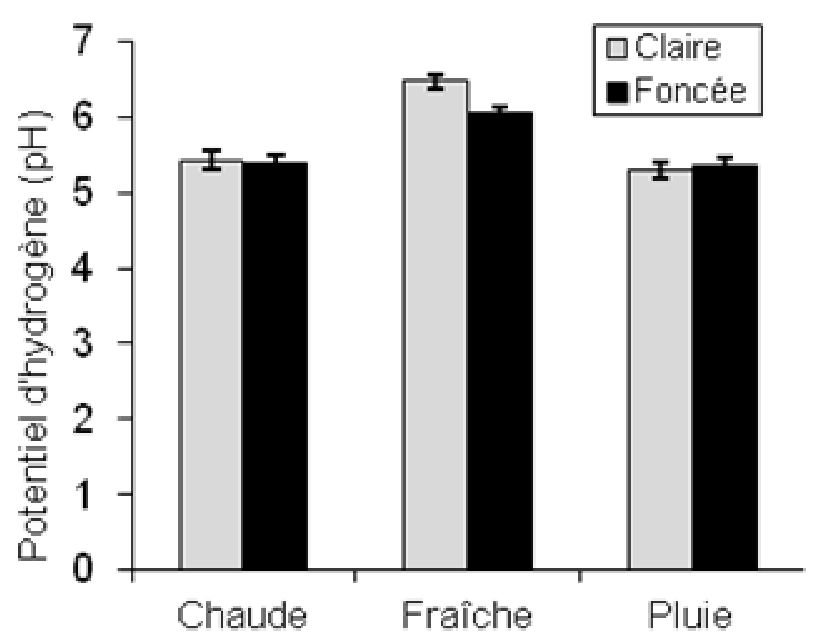

Figure 2: Influence de la période (sèche-chaude, pluies et sèche-fraîche) de saignée sur l'humidité (\%) de la gomme de $S$. setigera récoltée à Daoudi $(\mathrm{F}=71,61 ; \mathrm{p}=0,0000 ; \mathrm{n}=68)$.

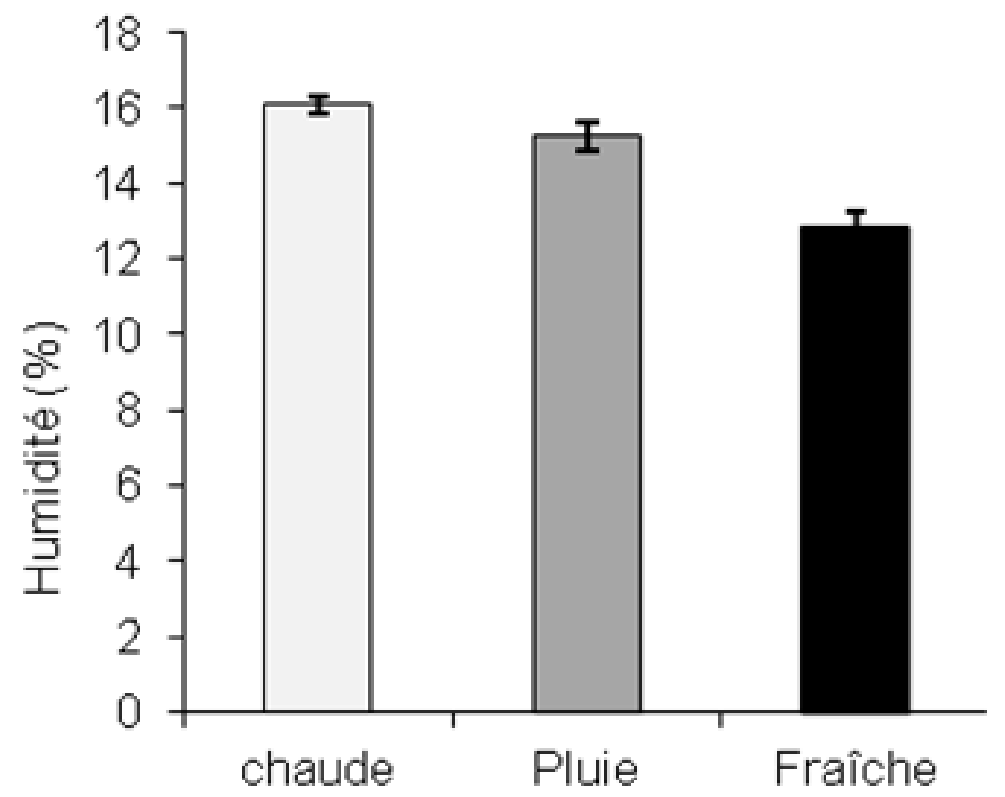

Figure 3: Interaction entre la période de saignée (sèche-chaude, sèche-fraîche et pluies) et la couleur de l'écorce (Claire, Foncée) sur le potentiel d'hydrogène $(\mathrm{pH})$ de la gomme de S. setigera récoltée à Malem Niani $(\mathrm{F}=3,92 ; \mathrm{p}=0,026 ; \mathrm{n}=60)$. 


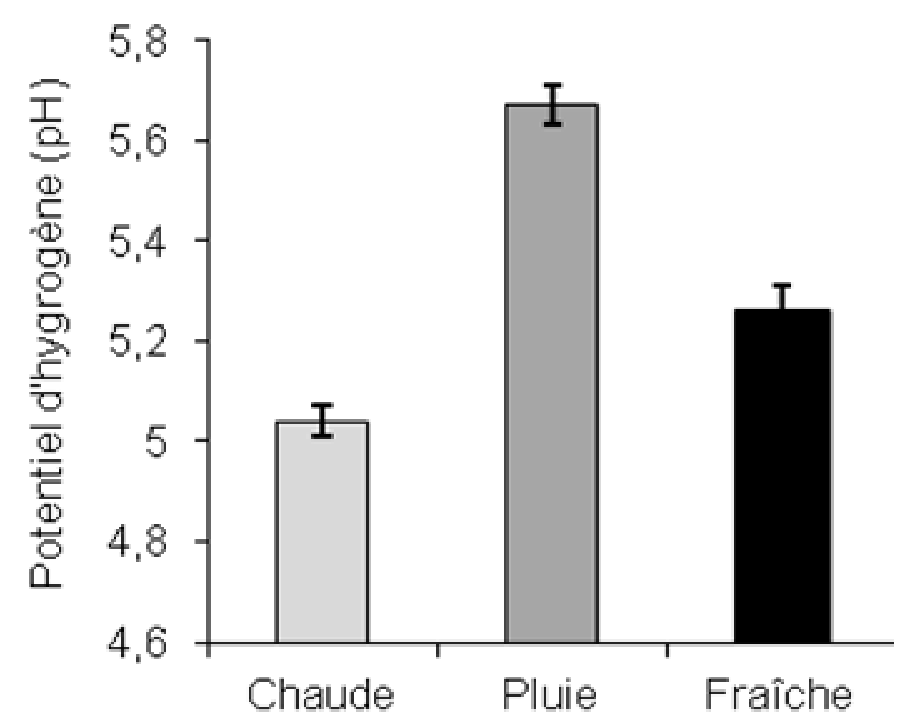

Figure 4: L'acidité de la gomme de S. setigera récoltée à Daoudi en fonction de la période (sèchechaude, pluie, sèche-fraîche) de saignée $(\mathrm{p}<0,0000 ; \mathrm{n}=68)$.

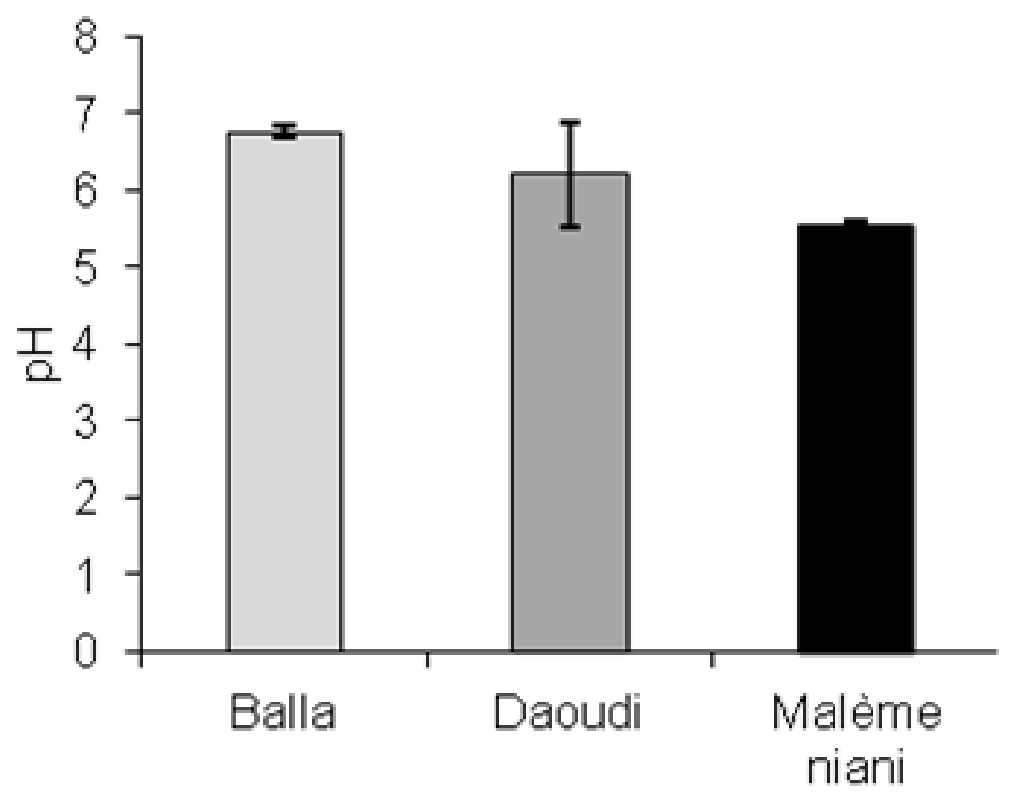

Figure 5: Potentiel hydrogène $(\mathrm{pH})$ de la gomme de $S$. setigera âgée d'une année et récoltée en période sèche-chaude à Balla, Daoudi et Malem Niani $(F=71,25 ; \mathrm{p}=0,0000 ; \mathrm{n}=71)$. 


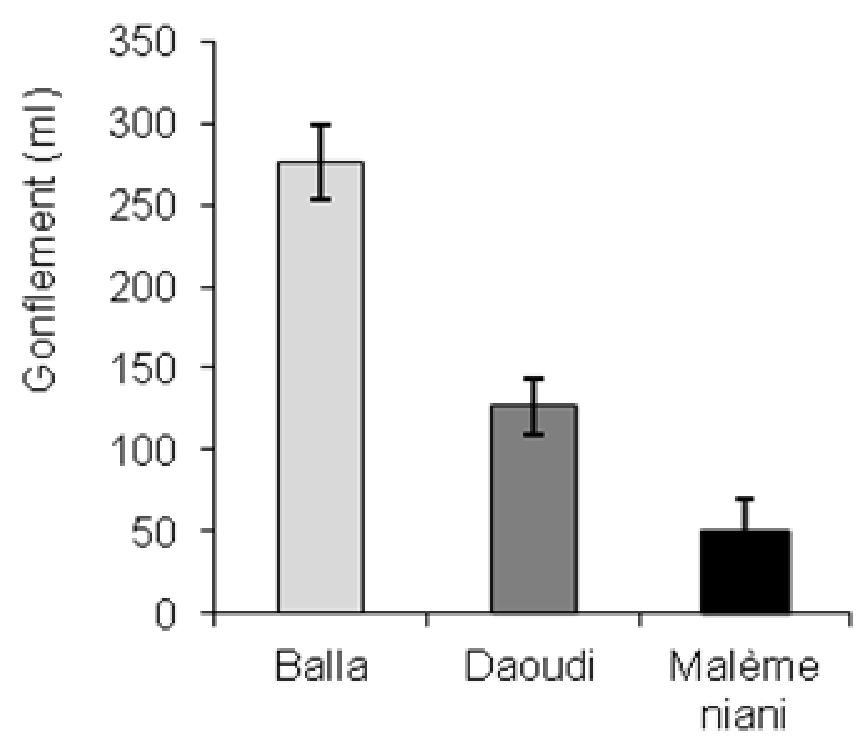

Figure 6: Gonflement de la gomme de S. setigera en fonction de la couleur (claire, foncée) de l'écorce et de la période de récolte (pluvieuse après saignée, pluvieuse après rafraîchissement, sèche-chaude après saignée, sèche-chaude après rafraîchissement), récoltée à Malem Niani $(\mathrm{F}=$ $22,90 ; \mathrm{p}=0,039 ; \mathrm{n}=94)$.

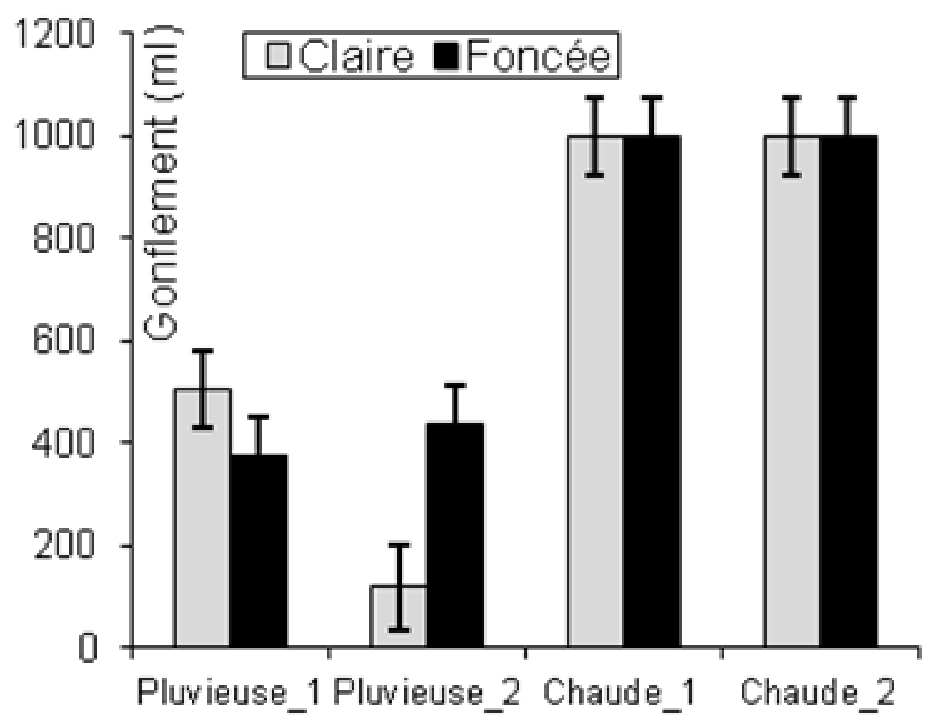

Figure 7: Gonflement de gommes de $S$. setigera âgées d'un an et récoltées à Balla, Daoudi et Malem Niani $(\mathrm{F}=30,56 ; \mathrm{p}<0,0000 ; \mathrm{n}=64)$. 


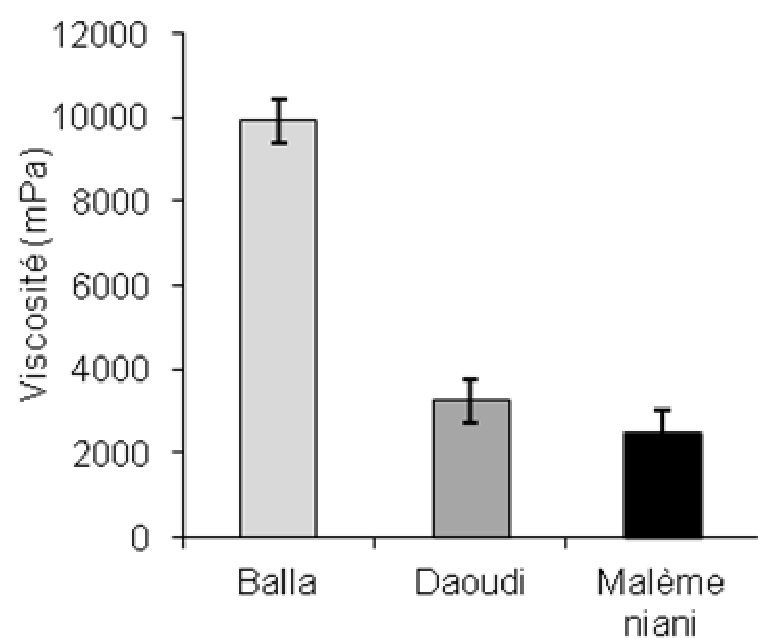

Figure 8: Influence du site (Balla, Daoudi, Malem Niani) sur la viscosité de la gomme de $S$. setigera $(F=62,84 ; p<0,0000 ; n=27)$.

\section{DISCUSSION}

L'humidité des gommes étudiées a varié entre 12,7 et $17,7 \%$ en fonction de la période de récolte, de l'origine ou de la couleur de l'écorce des arbres. Elle n'a cependant pas varié entre les provenances de gomme après un an de conservation. Des taux d'humidité de $13,5 \%$ à $17,4 \%$ ont aussi été rapportés par Lô (1996). Des taux d'humidité de 12 à $14 \%$ ont été publiés pour la gomme karaya indienne (S. urens) mais selon Le Cerf (1992), la gomme karaya est vendue en Inde avec 18 à $20 \%$ d'humidité.

La gomme mbepp, très hydrophile, est souvent le siège de développement de microorganismes (Lô, 1996) qui peuvent affecter sa qualité commerciale. Des actions allant dans le sens d'une meilleure maîtrise des techniques d'exploitation, de séchage et de stockage sont donc indispensables. Cet essai confirme par ailleurs l'hypothèse des populations selon laquelle les deux phénotypes produisent des gommes de qualités différentes (communication personnelle).

L'acidité de la gomme récoltée à Daoudi a varié en fonction de la période: elle a été plus élevée $(\mathrm{pH}=5,04)$ en période sèche-chaude qu'en période sèche-fraîche $(5,26)$ ou qu'en période pluvieuse $(5,67)$. A Malem Niani elle a été fonction de l'interaction entre la période de saignée et la couleur de l'écorce avec des $\mathrm{pH}$ variant de 5,36 à 6,45 . Ces valeurs sont similaires à celles obtenues par Verbeken et al. (2003) pour la gomme tragacanth dont le $\mathrm{pH}$ varie entre 5 et 6 , à celles de la gomme de Cochlospermum gossipium DC. avec des valeurs de 4,9-5,0 (Vinod et Sashidhar, 2010) et de la gomme de $S$. foetida avec un $\mathrm{pH}$ de 4 à 5 (Amit et al., 2008). Les valeurs moins acides de la gomme à Daoudi $(5,67)$ et à Malem Niani (6) peuvent s'expliquer par les conditions de stockage de la gomme à la température ambiante qui ont favorisé une perte d'acidité.

La période de récolte influence également l'acidité de la gomme. Les valeurs d'acidité obtenues au cours de cette étude durant toutes les périodes ont été très proches du pH normal (5). Des résultats semblables avaient déjà été obtenus dans ces mêmes sites (Daoudi : $\mathrm{pH}=4,97)$ et Bala $(\mathrm{pH}=5,35)$ par Lô (1996) et Lô (2005). Les gommes karaya d'origine indienne et sterculia d'origine africaine ont généralement des acidités variant 
entre 4 et 7 et entre 4,7 et 5,2 respectivement selon Lô (1996) et Verbeken et al. (2003).

Le gonflement de la gomme de Daoudi n'a pas varié suivant la période de saignée contrairement à la gomme de Malem Niani. A Daoudi, le gonflement a été de $1000 \mathrm{ml}$ et reste supérieur à la limite inférieure fixée sur le marché mondial $(60 \mathrm{ml})$ selon Lô (1996). Par contre, pour Malem Niani, le gonflement a été plus élevé en période sèche-chaude et en période sèche-fraîche $(1000 \mathrm{ml})$ et plus faible en période pluvieuse $(439,3 \mathrm{ml})$.

Lorsqu'on compare le gonflement des gommes obtenues après saignée et les gommes obtenues après rafraîchissement des saignées, il semble que le gonflement reste lié à la période de collecte et à la couleur de l'écorce (Figure 8). Cette différence du pouvoir gonflant entre les deux phénotypes est manifeste uniquement en période de pluies après rafraîchissement où il est plus élevé (437,5 ml) avec le phénotype foncé qu'avec le phénotype clair $(120,4 \mathrm{ml})$. D'après Lô (2005), la gomme récoltée en période sèchechaude et conservée 1 à 2 mois en conditions naturelles peut présenter des pouvoirs de gonflement supérieurs à $1000 \mathrm{ml}$. Nos résultats confirment ce fort pouvoir de gonflement évoqué par Lô (2005).

Ce pouvoir a aussi varié avec la durée et les conditions de conservation (Figure 7). Celui des gommes de Bala $(276 \mathrm{ml})$ et de Daoudi (126,54 ml) a chuté de façon significative, après un an de conservation en conditions naturelles. Cependant, cette baisse reste toujours dans la limite acceptable pour une gomme commercialisable sur le marché mondial.

Par contre, le gonflement de la gomme de Malem Niani a chuté de façon plus importante après un an de conservation pour atteindre $50,55 \mathrm{ml}$, valeur inférieure à la norme exigée dans l'industrie pharmaceutique (Lô, 1996). Le pouvoir gonflant de la gomme karaya diminue aussi en fonction de la durée de stockage. L'exposition à la chaleur et à un taux d'humidité élevé pourraient être les facteurs expliquant cette diminution avec le temps.

La viscosité de la gomme mbepp récoltée en période sèche-chaude et conservée durant un an a varié en fonction de son origine (Figure 9). Celle de Bala a été plus élevée (9913 mPa) que celle de Daoudi (2872 mPa). Ces résultats présentent un intérêt pour la commercialisation de la gomme mbepp, car pour une même utilisation, une plus faible quantité de gomme provenant de Bala sera nécessaire par rapport aux autres gommes, ce qui permettrait de faire une économie de produit. Selon Phillips (1998), la viscosité des dispersions de gomme varie en fonction de la catégorie mais s'étend de 0,10 à 0,40 Pas pour $0,5 \%$ à 10 Pas pour $3 \%$. II est possible que la différence de viscosité entre les échantillons soit liée à leur acidité: Bala $(\mathrm{pH}=6,75)$ le moins élevé, suivi par Daoudi $(6,20)$ et puis de Malem Niani (5,53). La viscosité est influencée par le $\mathrm{pH}$ de la solution: elle est maximale à $\mathrm{pH} 8,5$, seuil au dessus duquel les solutions ont tendance à être visqueuses (Lô, 1996). La différence de viscosité entre les échantillons pourrait s'expliquer par la présence d'impuretés (débris, sable, autres) car la gomme analysée n'était pas de la qualité Hand Pecket Selected (HPC) (Johnson et al., 2005).

La viscosité d'une solution de gomme varie en fonction de plusieurs autres facteurs tels que la granulométrie, la concentration en gomme, la température, l'ion calcium, le gradient de cisaillement, l'âge de la gomme (Babu et al., 2002a). La viscosité de la gomme de $S$. setigera après un an de conservation à la température ambiante était encore très élevée (Figure 9).

\section{REFERENCES}

Amit AC, Sushilkumar SP, Shajahan A, Gaurav S. 2008. Evaluation of Sterculia foetida Gum as Controlled Release Excipient. AAPS Pharm. Sci. Tech., 9(1): 197-204.

Anderson DMW, McNab CGA, Anderson CG, Brown PM, Pringuer A. 1985. 
Studies of uronic acid materials, Part 58: Gum exudates from the genus Sterculia (gum karaya). Int. Tree Crops J., 2: 174154.

Babu GVMM, Kumar NR, Sankar KH, Ram BJ, Kumar NK, Murthy KVR. 2002a. In vivo evaluation of modified gum karaya as a carrier for improving the oral bioavailability of a poorly water-soluble drug, Nimodipine. AAPS Pharm. Sci. Tech., 3(2): 55-63.

Babu GVMM, Prasad CDS, Murthy KVR. 2002b. Evaluation of modified gum karaya as carrier for the dissolution enhancement of poorly water-soluble drug nimodipine. Int. J. Pharm., 234: 117.

Bartlett MS. 1947. The use of transformations. Biom., 3: 39-52.

Bergeret A, Ribot JC. 1990. L'arbre nourricier en pays sahélien. Ministère de la Coopération et du Développement et la Fondation de la Maison des Sciences de L'homme, Paris.

Bhattacharya P, Joshi B, Hayat SF. 2003. An improved method of tapping gum from Kullu (Sterculia urens). Forest Trees and Livelihoods, 13: 187-196.

Dieng O. 1995. Etude sur le prélèvement et l'exploitation de la gomme de Sterculia setigera à Koumpentoum au Sénégal. Mémoire de Master of Sciences, Université Laval, Québec, Canada.

Dziezak JD. 1991. A focus on gums. Food TechnoI., 45: 116-132.

FAO. 1992. Foresterie en zones arides-Guide à l'intention des techniciens de terrain. http://www.fao.org/docrep/t0122f/t0122f 00.htm.

Henric J. 2001. Sterculia setigera et la gomme mbep. Le Flamboyant, 54.

Idris OHM, Williams PA, Phillips GO. 1998. Characterization of gum from Acacia senegal trees of different age and location using multidetection gel permeation chromatography. Food Hydrocolloids, 12: $379-388$.
Imeson A. 1992. Exudate gums. In Thickening and Gelling Agents for Food, Imeson A (ed). Chapman and Hall: London; 66-97.

Islam AM, Phillips GO, Sljivo A, Snowden MJ, Williams PA. 1997. A review of recent developments on the regulatory, structural and functional aspects of gum arabic. Food Hydrocolloids, 11(4): 493505.

Janaki B, Sashidhar RB. 1998. Physicochemical analysis of gum koundagogou (Cochlospermum gossypium) : a potential food additive. Food Chemistry, 61: 231236.

Johnson AD, Sy MS, Faye M. 2005. Etude de cas sur le produit naturel le Laalo mbepp au Sénégal. United States Agency for International Development (USAID/ EGAT) International Resources Group (IRG).

Karamalla KA, Siddig NE, Osman ME. 1998. Analytical data for Acacia senegal var. senegal gum samples collected between 1993 and 1995 from Sudan. Food Hydrocolloids, 12: 373-378.

King K, Richard G. 1993. The effect of gamma irradiation on guar gum, locust bean gum, gum tragacanth and gum karaya. Food Hydrocolloids, 6: 559-569.

Kovel Foundation and International Resources Group (IRG). 2005. Report on the gum karaya sub-selector in Andhra Pradesh, India. USAID.

Le Cerf D, Irinei F, Muller G. 1990. Solution properties of gum exsudates from Sterculia urens (karaya). Carbohy. Pol., 13: 375-386.

Le Cerf D. 1992. Propriétés physicochimiques de solutions et de dispersions d'un polysaccharide anionique: la gomme Karaya, exsudat d'arbre du genre Sterculia. Relation avec les propriétés fonctionnelles et applications. Thèse nouveau Doctorat, Université de Paris 06, Paris, France.

Lô M. 1996. Contribution à l'étude botanique et physico-chimique des gommes de Sterculia L. (Sterculiaceae): valorisation 
de la production de Sterculia setigera Del. et incidences socio-économiques au Sénégal. Thèse de Doctorat, Université Cheikh Anta Diop, Faculté de médecine et de pharmacie, Dakar (Sénégal).

Lô I. 2005. Caractères physico-chimiques de la gomme de Sterculia setigera (DEL.). Rapport de stage. Option: Industries chimiques et agroalimentaire. ESP/UCAD.

Phillips GO. 1998. Acacia gum (gum Arabic): a nutritinal fibre; metabolism and calorific value. Food Addit. Contam., 15: 251-264.

Séne A. 1994. Etude socio-économique des systèmes à parc dans le bassin arachidier : cas de Sterculia setigera et de Cordyla pinnata. Mémoire de confirmation, Institut Sénégalais de Recherches Agricoles, Centre National des Recherches Forestières, Sénégal.

Traoré T. 1983. Evaluation des potentialités et perspectives de développement de la gomme mbepp (Sterculia setigera): problèmes, débouchés. Mémoire de fin d'études, Ecole Nationale des Cadres Ruraux, Bambey (Sénégal).

Verbeken D, Dierckx S, Dewettinck K. 2003. Exudate gums: occurrence, production, and applications. Appl. Microbiol. Biotechnol., 63: 10-21.

Vinod VTP, Sashidhar RB, Sarma VUM, Vijaya SUVR. 2008. Compositional analysis and rheological properties of gum kondagogu (Cochlospermum gossypium): a tree gum from India. Journal of Agricultural and Food Chemistry, 56(6): 2199-207.

Vinod VTP, Sashidhar RB. 2010. Surface morphology, chemical and structural assignment of gum kondagogu (Cochlospermum gossypium DC.): an exudate tree gum of India. Indian Journal of Natural Products and Resources, 1(2): 181-192.

Vinod VTP, Sashidhar RB, Sreedhar B. 2010. Biosorption of nickel and total chromium from aqueous solution by gum kondagogu (Cochlospermum gossypium): A carbohydrate biopolymer. Journal of Hazardous Materials, 178: 1-3.

Whistler RL. 1993. Exudate gums. In Industrial Gums: Polysaccharides and their Derivatives, Whistler RL, Bemiller JN (eds). Academy Press: San Diego; 318-337. 